\title{
Stability of error distributions within tachistoscopic patterns'
}

\section{E. RAE HARCUM, THE COLLEGE OF WILLIAM AND MARY MELSON F. SMITH, UNIVERSITY OF RHODE ISLAND}

To investigate possible commonality of mechanisms in tachistoscopic pattern perception and in serial learning, we attempted to produce an isolation effect, frequently. found in serial learning, in the perceptual task. Reference markers were placed within binary visual patterns in some exposures, and for particular blocks of exposures reference lines were reproduced on the responding templates. In some exposures special precautions were taken to prevent fixational errors. The distributions of errors among elements never varied. Thus, the perceptual curve was stable in spite of the manipulation of certain extemal variables. The absence of an isolation effect was attributed to $O$ 's critical lack of foreknowledge about the locus of isolation.

The distribution of errors among elements in the reproduction of a tachistoscopic pattern is similar to the bowed serial-position curve of learning (Harcum, 1966). Therefore, Harcum $(1965,1966)$ hypothesized that the perceptual and serial-learning tasks had some mechanisms in common-viz., a serial processing of elements which in turn have been differentiated and internally organized. The present experiment tests this hypothesis.

Although the bowed serial-position curve is a reliable phenomenon of learning, it can be altered by factors which are apparently extrinsic to the task itself, as in the well-known isolation-effect. The curve of errors in pattern perception also appears to be similarly stable and general (Harcum, 1964). The present experiment investigates the stability of this function for binary patterns when three variables which are external to the

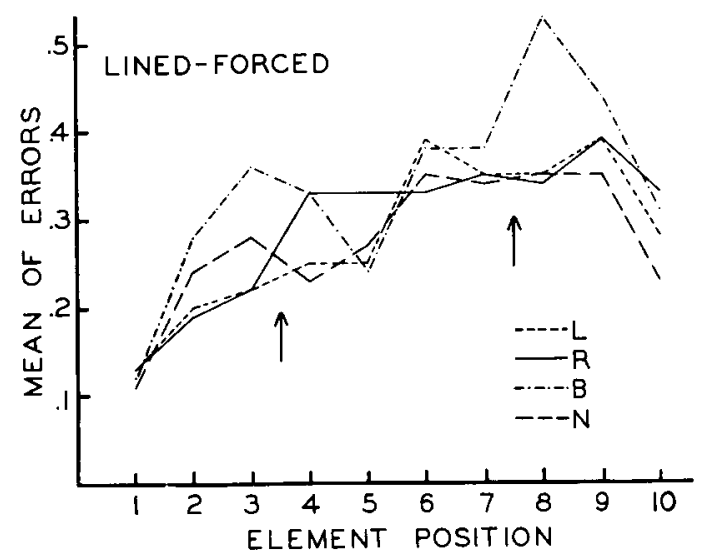

Fig. 1. Means of errors per exposure under "Lined" and "Forced" conditions. actual task are manipulated. If the perceptual data are altered in a way that might be expected for a seriallearning task, then the argument for similar mechanisms in the two tasks is supported. The argument is weakened if an external variable which cannot be part of the serial-learning process-such as the locus of ocular fixation-determines the distribution of errors in the perceptual task.

\section{Procedure}

Ten binary patterns of 10 blackened and open circles were exposed once each for .1 sec.in a Dodge tachistoscope, with fixation on center of pattern. Sixteen conditions were formed by all combinations of three variables. The first variable, of greatest interest, was the differential placement of reference lines on the stimulus pattern. In the "Left" and "Right" conditions a short vertical line was placed midway between the third and fourth, or seventh and eighth, circles from the left, respectively. "Both" and "Neither" conditions employed lines at both positions, or neither position, respectively.

The second variable was the placement of reference lines on the responding template. The "Lined" condition contained vertical lines between the third and fourth elements and between the seventh and eighth elements. The "Unlined" condition had no such lines.

The third variable was produced by an assessment of whether fixational accuracy affected the distribution of errors. Under "Forced" fixation, O could initiate the exposure only when a faint light was flashed at the fixation point, near which he had to be looking in order

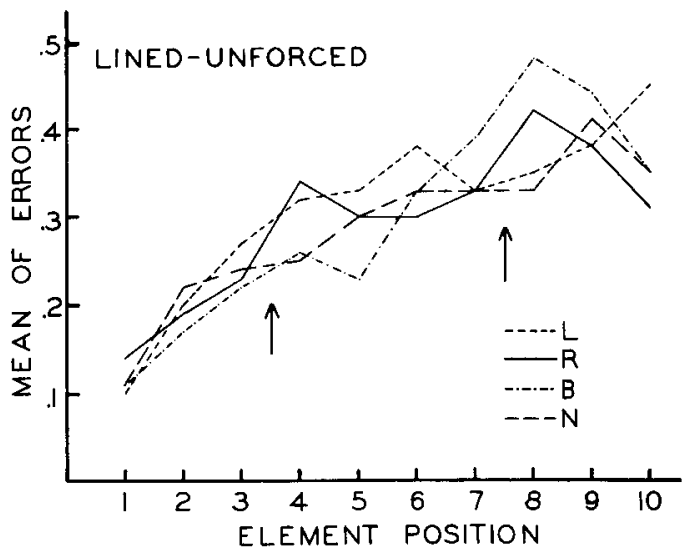

Fig. 2. Means of errors per exposure under "Lined" and "Unforced" conditions. 


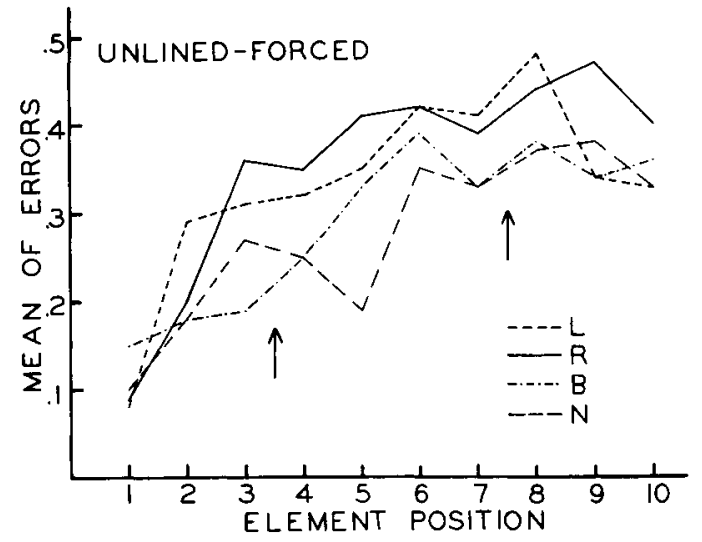

Fig. 3. Means of errors per exposure under "Unlined" and "Forced" conditions

to expose the target. "Unforced" fixation was maintained by instructions.

Each of the 12 Os served under all conditions, in counterbalanced sequences as far as possible. Os knew the fixation and recording-template condition before exposure, but not the stimulus-marker condition. After the exposure $O$ attempted to reprochuce the stimulus pattern onto the responding template. Each incorrect reproduction of an element counted as one error. Results

Means of errors per exposure for each element are shown in Figs. 1,2, 3, and 4, which permit a direct comparison of results for the four stimulus conditions. Locations of reference lines are indicated by arrows. The uniformity of the distributions of errors for all curves is obvious. Results of the individual Os also did not imply a consistent effect for any of the manipulated variables. Actually, the variables of template-lineation and fixation-forcing produce a result which would be expected from extraneous variables-viz., inflation of the variability. In Fig. 4 the most "natural" situation, with unlined template and unforced fixation, produced the least variable results.

\section{Discussion}

Except for the ubiquitous appearance of the bowed element-position curve, the results of this study are negative. Thus, we conclude that the bowed curve of perceptual errors is a stable phenomenon, resistant to effects of external variables such as were used in this experiment. Moreover, the skewness of the distribution of errors seems not to be an artifact of fixational error.

Since there is no evidence for an isolation effect in this experiment, the hypothesis is not supported by the predicted result. Although we do not know whether the present technique of isolation would actually have

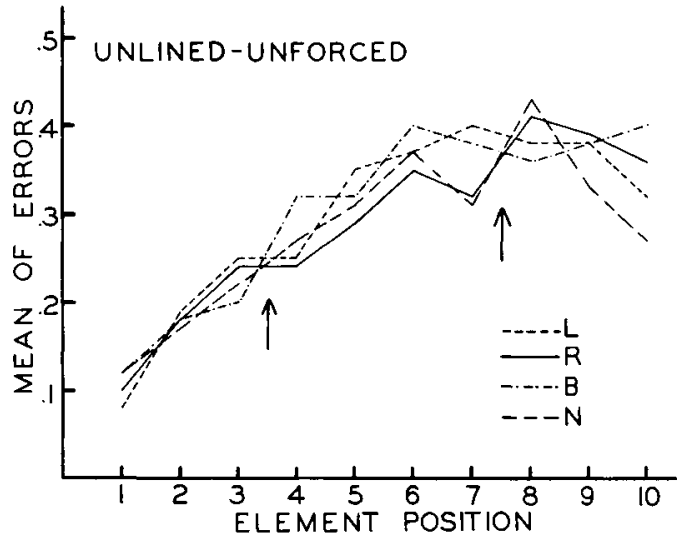

Fig. 4. Means of errors per exposure under "Unlined" and "Unforced" conditions.

affected a serial-learning curve, we doubt that our failure to find an isolation effect is due to the method of isolation. Other studies (e.g., Harcum, 1965) indicate that a critical condition for an isolation effect was not met in this experiment; a single exposure in tachistoscopic pattern perception is not likely to produce an isolation effect. Moreover, Saltz \& Newman (1959) failed to discover stronger association for an isolated item to the preceding stimulus item after only one trial of serial learning. Thus, as Harcum (1966) concludes, some temporal interval of foreknowledge of isolation is critical for an isolation effect in learning or in perception when item location must be reproduced. This commonality of negative results actually strengthens the argument for common mechanisms in terms of S's strategy of attending to the individual items.

\section{References}

Harcum, E. R. Reproduction of linear visual patterns tachistoscopically exposed in various orientations. Williamsburg, Virginia: The College of William and Mary, 1964.

Harcum, E. R. Pre-knowledge of isolation as a prerequisite for the isolation-effect. Psychon. Sci., 1965, 3, 443-444.

Harcum, E. R. Mnemonic organization as a determinant of errorgradients in visual pattern perception. Percept. mot. Skills, 1966, 22, 671-696 (Monograph Suppl. 5-V22).

Smith, N. F., \& Harcum, E. R. Perception of binary patterns with and without reference markers. Va. J. Sci., 1963, 14, 274. (Abstract)

Saltz, E., \& Newman, S. E. The von Restorff isolation effect: test of the intralist association assumption. J. exp. Psychol., 1959, $58,445-451$.

\section{Note}

1. This investigation was supported by a PHS research grant (HD 00207-06) from the Institute of Child Welfare and Human Development, Public Health Service. An abstract of the results has been published (Smith \& Harcum, 1963). 